\title{
The Duty-cycle of Gamma-ray Blazars: a New Approach, New Results
}

\author{
S. Vercellone*, S. Soldi ${ }^{\dagger}$, A.W. Chen ${ }^{* * *}$ and M. Tavani ${ }^{\ddagger * *}$ \\ ${ }^{*}$ IASF-CNR Sezione di Milano, Via Bassini 15, 20133 Milano - Italy \\ ${ }^{\dagger}$ ISDC, Chemin d'Ècogia 16, 1290 Versoix - Switzerland \\ ** CIFS, Viale Settimio Severo 63, Torino - Italy \\ IASF-CNR Sede di Roma, Via del Fosso del Cavaliere 101, Roma - Italy
}

\begin{abstract}
We study several properties of blazars detected in the gamma-ray energy range by comparing the EGRET sources with a sample of radio blazars which can be considered possible gamma-ray candidates. We define three classes: non-gamma-ray blazars, blazars with quasi-steady gamma-ray emission, and gamma-ray blazars with substantial activity level. By combining the information of detected and candidate AGNs, we characterise the blazar activity, including the discovery of a region of consistency between the gamma-ray flaring duty-cycle and the recurrence time between flares. We also find a possible relation between the activity index of FSRQs and their black hole mass.
\end{abstract}

\section{INTRODUCTION}

Among Active Galactic Nuclei (AGN), blazars show strong flux variability at almost all frequencies of the spectral energy distribution (SED). The EGRET instrument on-board of CGRO detected, above $30 \mathrm{MeV}$, blazars as a class of $\gamma$-ray sources [3], identifying 67 objects, and detecting 27 candidates. Gamma-ray blazars are characterised by high variability on different time-scales, from one day (e.g. PKS 1622-297) to one month (e.g. PKS 0208-512). This large spread in time variability and the sparse coverage obtained by EGRET make it difficult to quantify parameters such as the duty-cycle (i.e. the fraction of time spent in a flaring state) and/or the characteristic time-scale of $\gamma$-ray activity for this class of sources.

A preliminary result was obtained by [6], based on the Second EGRET Catalogue assuming that the link between the radio and the $\gamma$-ray emission can be used to derive the $\gamma$-ray luminosity function. Under these assumptions, they found that a duty-cycle $\zeta=0.03$ is consistent with the data. One weakness of this analysis is that the radiogamma relationship is highly uncertain.

The aim of this Paper is to summarize a recent result on the estimate of the blazar duty-cycle and $\gamma$-ray variability [9]. 


\section{THE BLAZAR $\gamma$-RAY VARIABILITY}

In order to investigate the blazar $\gamma$-ray duty-cycle, we analysed the Third EGRET Catalogue (3EG) AGN sample, looking for recurrent activity. For each source we considered the number of viewing periods (VPs) during which it was close enough to the pointing direction $\left(<40^{\circ}\right.$, the FOV radius) that its exposure for that VP was greater than zero. For each source we calculated the Exposure $(E X P)$, defined as the sum of the exposures during each single viewing period.
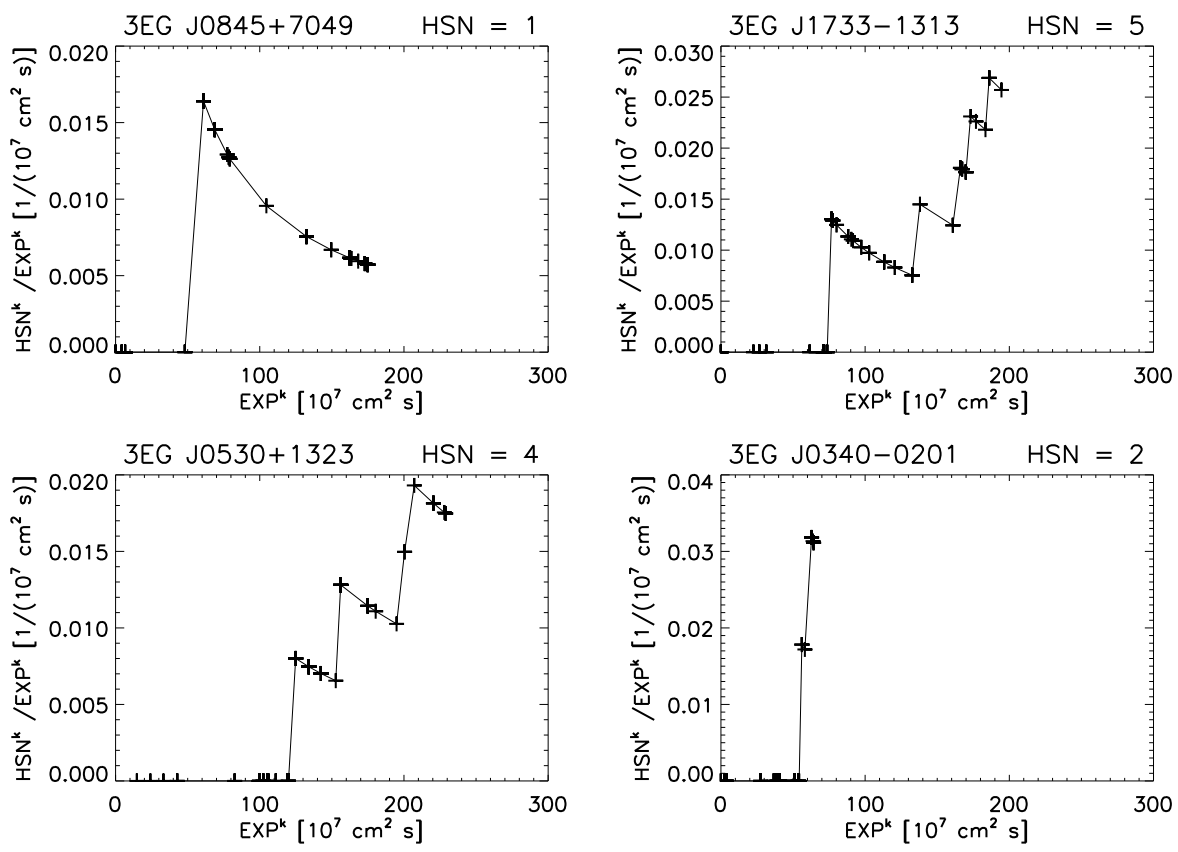

FIGURE 1. Four different examples of blazar $\gamma$-ray activity as a function of exposure. Top-left panel: 3EG J0845+7049 with $H S N=1$ despite its high exposure; Top-right panel: 3EG J1733-1313 which, for a similar exposure, has $H S N=5$; Bottom-left panel: 3EG J0530+1323 which showed a possible periodicity in its high-state status, and Bottom-right panel: 3EG J0340-0201 which has a very low exposure $\left(\sim 64 \times 10^{7} \mathrm{~cm}^{2} \mathrm{~s}\right)$, but showed 2 high-states in a few observations. Crosses show the value of the discrete $\psi$ for each VP containing the source.

In order to estimate the $\gamma$-ray activity level, we computed the number of times that a source was in a high-state (HSN) i.e. when the flux of the $i$-th VP was greater than a given threshold. We establish that a source is in a high-state when 1) the flux of the $i$-th VP is in excess of 1.5 times the mean flux of the source, and 2) the 1- $\sigma$ uncertainty of the measurement at the high state is less than the deviation of the measurement from the mean. The mean flux is defined as the weighted mean of all the detections and upper limits, assuming the weight $w(\sigma)=1 / \sigma$, where $\sigma$ is the uncertainty of the measurement for detections, while $\sigma=U L$ for the upper limits. If two high-states were less than 2 weeks apart (the typical duration of an EGRET pointing), we gathered them into a single high-state coincident with the first one.

Given EXP and HSN, we computed an activity index ( $\psi$ ) of each AGN as follows:

$$
\psi_{\mathrm{src}}=H S N_{\mathrm{src}} \times\left[E X P_{\mathrm{src}}\right]^{-1}\left[\mathrm{~cm}^{-2} \mathrm{~s}^{-1}\right] .
$$


This index provides an estimate of blazar $\gamma$-ray activity, weighted by the exposure. Sources with high $\psi$ have a $\gamma$-ray activity which is highly variable. Sources with $\psi=0$ do not show significant flux variability.

We analysed the behaviour of each single source having $\psi \neq 0$ during its time history. Fig. 1 shows the discrete $\psi$ for some EGRET sources as a function of EXP. We show four different examples of activity behaviour: 1) 3EG J0845+7049, with a single $H S N=1$ despite its large exposure; 2) 3EG J1733-1313, which, for a similar exposure,

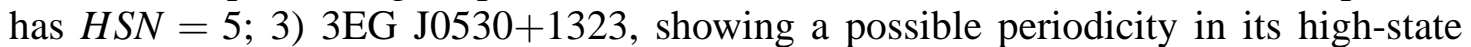
status, and 4) 3EG J0340-0201, which has a very low exposure ( $\left.\sim 64 \times 10^{7} \mathrm{~cm}^{2} \mathrm{~s}\right)$, but showed 2 high-states in a few observations.

Sources that have $H S N=1$ (e.g., 3EG J0845+7049) show a rise of $\psi_{s r c}^{k}$ followed by a decay after reaching a single high state, while sources that have high HSN (e.g. $3 \mathrm{EG} \mathrm{J} 0530+1323$ ) show a saw-tooth pattern, corresponding to alternately increasing/decreasing of $\psi_{\mathrm{src}}^{k}$.

We distinguish sources for which the activity level $\psi=0$ (population $A, 36$ per cent), and the ones having $\psi \neq 0$ (population $B, 64$ per cent). A remarkable difference appears in the two populations when we compare the ratio between flat-spectrum radio quasars (FSRQs) and BL-Lac objects (BLs). Population A is almost equally divided between FSRQs and BLs ( $\sim 60$ and $\sim 40$ per cent, respectively), while Population B is dominated by FSRQs $(\sim 80$ per cent). FSRQs are generally more variable and more luminous in the $\gamma$-ray energy band than BLs, which could explain the higher fraction of these sources in Population B. A possible correlation is found between the activity level and the black hole mass, with a probability $P_{\text {rand }}$ of a randomly distributed sample of objects on the order of $\sim 10^{-2}$. We suggest that the use of the $\psi$ statistic is more robust than the use of the HSN, since the latter is not weighted by the EGRET coverage.

\section{THE BLAZAR GAMMA-RAY DUTY-CYCLE}

We develop a simplified model of EGRET blazar activity that yields rough, but quantifiable, estimate of physical parameters of interest. In this simplified model, we make several assumptions:

1. that all of the EGRET blazars exhibit the same basic behaviour. While observationally unjustified, this assumption is necessary to model the average behaviour.

2. that only those blazars detected by EGRET are part of the population of interest.

3. that the behaviour of all EGRET blazars can be characterised by a simple model with only two free parameters, the duty-cycle, $\chi$, and the characteristic time-scale, $T$. Each blazar will spend a period of time whose average length is $T$ at a low flux level (off) before emitting a $\gamma$-ray flare of duration $\tau$ at a high flux level (on), then returning to a low level. The duty-cycle is then defined as the fraction of time spent in the on state:

$$
\chi=\frac{\tau}{\tau+T} .
$$

Note that all blazars have the same characteristic time-scale $T$, but the duration of each individual quiescent periods is drawn from a Poisson distribution with mean 
$T$.

We explored the parameter space of $(\chi, T)$. For each pair of $\chi$ and $T$ under consideration, we generated 100 sets of 67 simulated light curves covering the entire time interval of EGRET Cycle 1 to 4 (1620 days), one for each blazar. The time sequence of onoff states was determined by drawing the durations of the off states from a Poisson distribution with mean $T$. The durations of the on states were fixed at $\tau$. To obtain the observed fluxes, we used a bootstrap procedure. Each light curve was compared with the observation history of the corresponding AGN. If a given viewing period (VP) coincided with an on state, the observed flux was randomly drawn from the distribution of all fluxes detected from all EGRET blazars during a single VP. Otherwise, the observed flux was randomly drawn from the distribution of all upper limits (i.e. non-detections) at EGRET blazar positions during a single VP. For each AGN the flux values assigned to each VP were used to calculate its $H S N$ (and therefore its categorisation as Population A or B) in the same manner as the actual EGRET blazar HSN. The result is 100 sets of simulated blazar light curves, each with a ratio $R_{\mathrm{AB}}=$ \#Pop.A/(\#Pop.A +\#Pop.B). Thus for each pair of values of $(\chi, T)$, we have obtained a distribution of 100 simulated values of $R_{\mathrm{AB}}$, each with a mean and variance. Therefore, we are able to define a confidence region of $(\chi, T)$ pairs whose mean values of $R_{\mathrm{AB}}$ are within one standard deviation of the observed value $R_{\mathrm{AB}}=24 / 67=0.36$. For values of $R_{\mathrm{AB}}$ close to the observed value, the simulations give a standard deviation of 0.05 . We ran 100 simulations each for 390 $(\chi, T)$ pairs, ranging from $0<T<450$ days and from $0<\chi<1$. Fig. 2 shows the results. Points in Fig. 2 represent the mean value of $R_{\mathrm{AB}}$ for each value of $T$ and $\chi$.

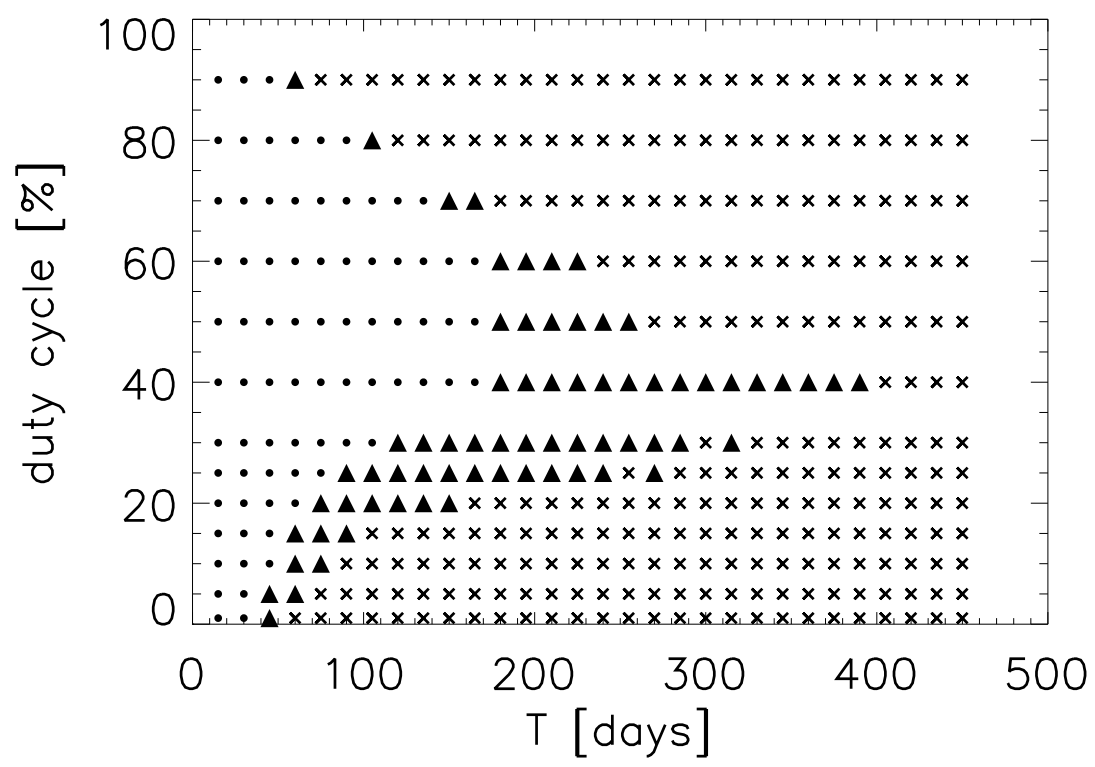

FIGURE 2. Mean ratio $R_{\mathrm{AB}}=$ \#Pop.A/(\#Pop.A + \#Pop.B) from simulations of EGRET -detected blazars only.

The filled triangles represent those ratio values between $0.31 \leq R_{\mathrm{AB}} \leq 0.41$, small filled 
circles represent $0.0 \leq R_{\mathrm{AB}}<0.31$, while crosses represent $0.41<R_{\mathrm{AB}} \leq 1.0$. Taking into account our simplifying assumptions, including the restriction that the observed EGRET blazars form the entire population of $\gamma$-ray emitting blazars, the only region consistent with the observations is the region where $0.31 \leq R_{\mathrm{AB}} \leq 0.41$. The region with high $R_{\mathrm{AB}}$ produces too few flaring sources, while the region with low $R_{\mathrm{AB}}$ produces too many. This implies that the true characteristic time-scale $T$ lies between 50 and 400 days, the duty-cycle $\chi$ becoming more extreme for lower values of $T$. Note that for the Stecker \& Salamon duty-cycle of 0.03 the characteristic time-scale $T$ would be on the order of 50 days.

If we should take into account a wider sample of $\gamma$-ray blazar, by including radio blazars emitting at a level of $250 \mathrm{mJy}$, we would obtain that the low $R_{\mathrm{AB}}$ region to remain excluded, because adding more sources is unlikely to reduce the absolute number of Population B sources predicted, which is higher than the number observed. On the other hand, the $(\chi, T)$ pairs in the high $R_{\mathrm{AB}}$ region may now be consistent with the EGRET data. Therefore, the region in $(\chi, T)$ space where formerly the $R_{\mathrm{AB}}$ was consistent with the observed value now forms a lower bound to the possible values of $T$ for a given value of $\chi$.

\section{THE BLACK HOLE MASS - ACTIVITY RELATIONSHIP}

Recently, [2] estimated the black hole mass for 36 EGRET AGNs from their optical continuum luminosity; they claim a positive correlation (significance level 96.6 per cent) between the maximum $\gamma$-ray source luminosity and the black hole mass.

We look for a possible correlation between the $\gamma$-ray maximum luminosity, $L_{\gamma}^{\max }$, and the black hole mass, $M_{\mathrm{BH}}$, obtained by adopting the black hole masses reported in [8] for all the EGRET blazars except for 3C 273, whose $M_{\mathrm{BH}}$ was taken from [5]. The correlation is absent when considering the whole sample (Spearman statistic: $\rho=0.44$, and $P=0.15$ ), and becomes marginal if we take into account the FSRQs only ( $\rho=0.60$, and $P=0.08$ ). The relation between luminosity and black hole mass

is, approximately, $L_{\gamma} \propto M_{\mathrm{BH}}^{0.9}$ for FSRQs, consistent with the predicted relation by [1], although their assumptions ( $\gamma$-ray emission is isotropic radiation produced by the steady and Eddington-limited accretion without considering the beaming effect) do not agree with recent results on $\gamma$-ray blazars [4]. When analysing the possible correlation between the activity level, $\psi$, and the maximum $\gamma$-ray luminosity, we find no correlation $(\rho=$ 0.14 , and $P=0.39$ ). We found a possible correlation between the activity index $\psi$ and $M_{\mathrm{BH}}$. Fig. 3 shows our results based on ten blazars with non-zero $\psi$ and the black hole mass estimation. The Spearman correlation parameters turn out to be $\rho=0.76$ and $P=0.011$. This possible correlation seems to indicate that more massive BHs can induce higher $\gamma$-ray activity, although our sample is too small to draw a firm conclusion. More reliable $\mathrm{BH}$ mass estimates of a larger blazar sample will be crucial in verifying the existence of this correlation. 


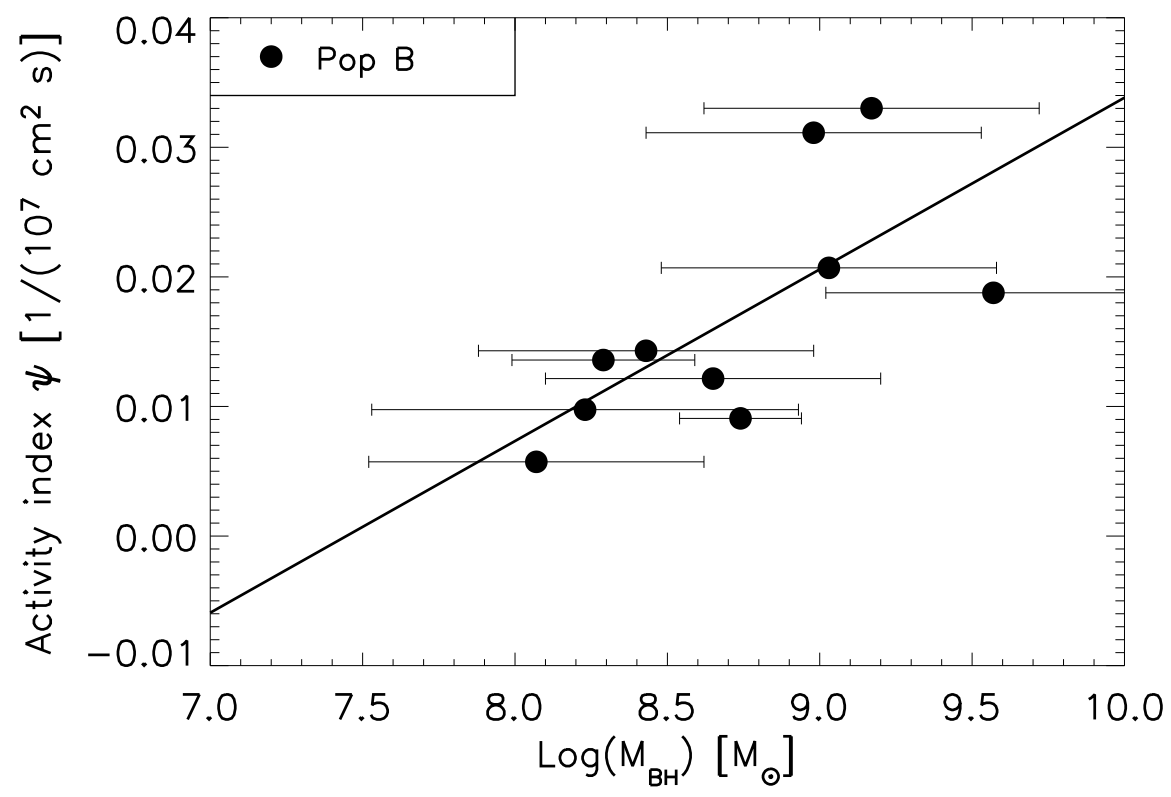

FIGURE 3. $\gamma$-ray activity $(\psi)$ vs. black hole mass $\left(M_{\mathrm{BH}}\right)$ for EGRET Pop. B blazars. A marginal correlation is present ( $\rho=0.76$ and $P=0.01$, Spearman statistic). The solid line represents the best fit line, $\log (\psi)=0.013 \times \log \left(\mathrm{M}_{\mathrm{BH}}\right)-0.099$. Horizontal bars represent the typical black hole mass estimate uncertainty as reported by [7].

\section{CONCLUSIONS}

The results presented here summarize a more detailed analysis presented in [9] where we discuss the implications on the duty-cycle estimate of a sample of candidate $\gamma$-ray AGNs extracted from recently published radio catalogues. A firm estimate of the dutycycle will be a scientific goal of future $\gamma$-ray missions such as AGILE and GLAST. Their wide FOV $\left(\sim 60^{\circ}\right)$ will allow monitoring of a large number of AGNs for each pointing on long time-scales. For the time being, an extensive black hole mass estimation by means of optical spectroscopy on FSRQs and BL Lac objects will allow us to better understand the relation between the central engine and the $\gamma$-ray activity.

\section{REFERENCES}

1. Dermer C. D., Gehrels N., 1995, ApJ, 447, 103

2. Fan Z., Cao X., 2004, ApJ, 602, 103

3. Hartman R. C. et al., 1999, ApJS, 123, 79

4. Jorstad S. G. et al., 2001a, ApJS, 134, 181

5. Kaspi S., Smith P. S., Netzer H., Maoz D., Jannuzi B. T., Giveon U., 2000, ApJ, 533, 631

6. Stecker F. W., Salamon M. H., 1996, ApJ, 464, 600

7. Vestergaard M., 2004, ASP Conf. Series, Vol. 311, p. 69

8. Woo J., Urry M.C., 2002, ApJ, 579, 530

9. Vercellone S., Soldi S., Chen A.W., Tavani M., 2004 MNRAS, 353, 890 